\title{
Las ciencias sociales en sus construcciones y controversias: Proximidades y vínculos con y desde lo social
}

\author{
Adela Bork Vega ${ }^{1}$ \\ Escuela de Trabajo Social e Instituto de Historia, Pontificia Universidad \\ Católica de Valparaíso, Chile
}

Resumen: Interrogar el estado de la cuestión referida a las ciencias sociales y la relación a los cambios culturales, sigue siendo un desafío significativo si la pregunta se configura cuestionando las dis-continuidades e influencia que tiene el discurso y práctica científica al interior de una determinada sociedad. El hacer un punto de inflexión sobre las ciencias sociales, potencialmente contribuye a identificar de manera contradictoria la relación y no relación entre un campo de influencia de esta entidad sobre las formas sociales que condicionan los cambios desde un enfoque cultural (BRAUDEL, 1979). En este artículo se releva por lo mismo, la persistencia y resistencia de aquellas formas lineales y binarias que tienden a explicar lo social y la poca porosidad para visualizar los movimientos como aquellos intersticios producidos a nivel de los sujetos, las instituciones y las propias arquitecturas de las disciplinas que nutren la configuración de las ciencias sociales, próxima a la definición de universos de comprensión-explicación (WALLERSTEIN, 2009).

Palabras claves: Larga duración; Temporalidades; Mentalidades.

Título: As ciências sociais em suas construções e controvérsias: Proximidades e vínculos com e desde o social

Resumo: Interrogar o estado da questão relacionada às ciências sociais e a relação com as mudanças culturais permanece um desafio significativo se a pergunta se configura questionando as dis-continuidades e a influência que tem o discurso e prática científica no interior de uma determinada sociedade. O fazer um ponto de inflexão sobre as ciências sociais, potencialmente contribui a identificar de maneira contraditória a relação e não relação entre um campo de influência dessa entidade sobre as formas sociais que condicionam as mudanças a partir de uma abordagem cultural (BRAUDEL, 1979). Neste artigo, destacamos a persistência e a resistência daquelas formas lineares e binárias, que tendem a explicar o social e a baixa porosidade para visualizar os movimentos como aqueles interstícios produzidos a nível dos sujeitos, das instituições e das próprias arquiteturas das disciplinas que nutrem a configuração das ciências sociais, próxima à definição de universos de compreensão-explicação (WALLERSTEIN, 2009).

Palavras-chave: Longa duração; temporalidades; mentalidades.

Abstract: Questioning the state of the question related to the social sciences and the relationship to cultural changes, remains a significant challenge if the question is configured by questioning the differences and influence that the discourse and scientific practice has

\footnotetext{
${ }^{1}$ Doctora en Sociología por Universidad Católica de Lovaina, Bélgica. Profesora Titular Pontificia Universidad Católica de Valparaíso - Chile (PUCV). Escuela de Trabajo Social e Instituto de Historia. Orcid: http://orcid.org/0000-0001-8503-133X
}

E-mail: adela.bork@pucv.cl 
within a given society. Making a turning point on the social sciences, potentially helps to identify in a contradictory way the relationship and not relantioship, between a field of influence of this entity on the social forms that condition changes froma cultural approach (BRAUDEL, 1979). In this article, the persistence and resistance of those linear and binary forms, that tend to explain the social and the low porosity to visualize the movements as those interstices produced at the level of the subjects, the institutions and the architectures themselves are highlighted the disciplines that nurture the configuration of social sciences, close to the definition of undestanding-explanation universes (WALLERSTEIN, 2009).

Keywords: Long duration; Temporalities; Mentalities.

...miren entonces caer las últimas cataratas las derrumbadas olas, el azul del amor precipitándose...

Zurita

\section{Perspectiva compartida}

Las ciencias sociales durante un largo período, tuvieron un desarrollo de carácter incremental, sin mayores variaciones, haciendo de su discurso y práctica un continuo que cumplía con ciertos objetivos para los fines que éstas se habían configurado (LAHIRE, 2016). Sin embargo, por propia auto comprensión que define a las ciencias en su carácter de revisión crítica y sistemática, se genera niveles de saturación y giros en los cuales la dimensión epistémica mueve los límites en los cuales se definían a partir de fronteras y clausuras (BERTHELOT, 2000).

En este proceso de saturación para algunos autores y/o de giros en la producción de conocimientos, se observa como en esta "crisis paradigmática", uno de los elementos que contribuye directamente es la manera como la dimensión cultural incide directamente en las formas de variabilidad e incertidumbre del saber (WALLERSTEIN, 2009). Los cambios sucedidos en las sociedades, en momentos veloces y en momentos lentos con ciertas resistencias e inercias, son factores complejos en la manera como se advierte esta relación entre ciencia y sociedad.

Pareciera que esta relación es obvia y que no precisa de mayor detención, empero, se constata en algunos apreciaciones empíricas, que en forma similar a la desconexión entre las instituciones y los sujetos, se produce de una manera paradojal y no aquilatada suficientemente, una distancia entre la necesidad del conocimiento a partir del estado que presenta un determinado colectivo social.

Si bien, esta lejanía entre entidades claves como es la ciencia y la sociedad es un fenómeno que se expresa a nivel global, es dable interrogarse para el caso de la región latinoamericana, considerando alguno procesos que han caracterizado la región. La persistencia de las desigualdades en las diversas esferas de la vida social, como también en 
las formas de gestionar los saberes múltiples, son parte de una re-significación de esta relación entre conocimiento y sociedad necesaria de tener en cuenta, si se considera que ambas propician formas de gestar y/o promover la vida social.

Este artículo, propone una reflexión desde una perspectiva socio-histórica la construcción disciplinaria de las ciencias sociales contemporáneas, con el fin de que se puedan advertir -sintéticamente por lo que implica un artículo-, aquellos movimientos que inciden directamente en las formas de explicar lo social no como un todo homogéneo, sino más bien como formas sociales que se construyen orgánica e inorgánicamente. Esta manera, limitada por cierto, posibilita analizar y estimar que los procesos culturales que se orientan al cuestionamiento y a la promoción de mentalidades, pueden ser explicados en claves complejas y/o bien, en una suerte de continuo canónico que ratifica la distancia ya mencionada entre sociedad y ciencia.

A la perspectiva socio-histórica que aquilata lo social bajo una lógica de larga duración, se incorpora las derivadas a nivel de mentalidad y como éstas pueden aportar a los saltos cualitativos en los cuales se construyen las disciplinas contenidas y definidas como ciencias sociales.

En una síntesis reflexiva, se logran advertir aquellos desafíos que ambas entidades precisan enfrentar en lógicas de sinergia y con apertura contradictoria, evitando lo endógeno y mecánico que se orienta a la clausura del pensamiento científico y social, como también a las prácticas de los sujetos. Por antonomasia, sociedad y ciencia se asocian a movimientos y a pluralización, alejando lo que regularmente se presenta como racionalidades "científicas", sin implicancias en la formación de un pensamiento cultural.

\section{La dimensión de la larga duración o el despliegue de las temporalidades}

En la tradición de la historiografía crítica, los aportes de lo que se define como historia mínima, ocupan un lugar fundamental para establecer los vínculos o no vínculos que posiciona por lo mismo, la trayectoria y/o el devenir como flujo del y en el tiempo, evitando la cosificación y lo situado en formas homogéneas y autorreferenciales.

Los trabajos de Jelin (2002), y Goicovic (2019) a nivel de la región latinoamericana que densifican y prolongan lo trabajos de Braudel (1979), Wallerstein (2009), Hobsbawm (2013) -entre otros-, facilitan la mirada desde los giros y/o rupturas para incidir en la construcción de los fundamentos disciplinarios. Las temporalidades por lo mismo, mueven los ejes endógenos y permiten las aperturas para dimensionar aquello definido como propio en una lectura global y compartida. Al mismo tiempo, si agregamos una nueva complejidad, como la larga duración en esta forma de apreciar las temporalidades, se construye con una orientación para evitar una lectura fragmentada de los movimientos históricos, saliendo del clásico lugar de simplificación circunstancial cuando decimos: lo acontecido es un antes y un 
después, o bien, cuando frente a cualquier transformación se hace referencia a que adquiere una dimensión histórica. En ambos casos, se aprecia lo que algunos autores definen como simplificación de lo social o presentismo social (BYUNG-CHUL, 2016; LECHNER, 2002), orientado a quitar espesura a la vida socio-política y/o bien a des-materializar los discursos como prácticas que denotan la actividad humana como trabajo incesante para la configuración de la vida en común (ARENDT, 1995).

Braudel (1979), en el desarrollo de la categoría de larga duración, puede orientar la reflexión crítica a partir de sus aportes a través de la categoría de variante e invariantes trabajadas para revisar lo referido en materia de las ciencias sociales. Esta manera binaria y dicotómica que, si bien, es criticada a nivel de los discursos que circulan sigue teniendo presencia en las prácticas desplegadas a partir de aquello definido como los núcleos disciplinarios que orientan las racionalidades científicas en las cuales se fundamentan, como también en lo que la tradición de la ciencia ha buscado a través del tiempo: prestigio y legitimidad. Ambas dimensiones han privilegiado un tipo de producción científica, que en momentos hace perder el valor y existencia de la ciencia, como una actividad orientada a la sociedad en el enfrentamiento de aquello no comprensible, aquello problemático, como también aquello que por su expresión de ruptura, no logra ser accedido de forma directa. Una suerte de trabajo científico, más orientado a la propia trayectoria y productividad de quienes lo cultivan, que por las exigencias y dilemas en los cuales se ven envueltos.

De manera complementaria, Burke elabora una reflexión orientada a comprender como se logra y se llega a valorar la historia cultural, en distintos movimientos de lo que ha sido la historiografía en su largo devenir (BURKE, 2019). Este historiador, afirmaba como la premura en definir el tiempo en forma lineal, hacía surgir la idea de lo nuevo como un ámbito irreconocible y de carácter principal, casi cercano a lo esencial, siendo en su opinión, una especie de distorsión ya que lo nuevo en una dimensión de larga duración, es evidentemente relativo considerando que inclusive en la línea de tiempo que ordena los evento de manera cronológica, excede al nacimiento de las diversas disciplinas identificadas a la zona de pertenencia de las ciencias sociales.

Bajo esta lógica, las temporalidades adquieren un sello o impronta que va moldeando la actividad de las ciencias sociales, tanto en el contenido como en su proceder, es decir, forma y fondo van sufriendo el impacto de la sociedad sobre la ciencia. Para algunos autores, aquello que en su momento distinguía a la ciencia, va perdiendo los contornos habituales y, queda atrapado en las exigencias de los modelos económicos y político de la sociedad actual. En palabras de Wallerstein (2009), la ciencia como sistema-mundo, re-acomoda el discurso, la práctica y los horizontes con los cuales se presenta social y culturalmente.

Lo anterior, conlleva a un punto relevante a la hora de posicionar a las ciencias sociales en los nuevos escenarios sociales, políticos, económicos y culturales. La dimensión discursiva de las ciencias sociales se enfrenta a una disyuntiva paradojal, de una parte, persistir en sus formas de quehacer asociadas a un saber-poder, orientada a una hegemonía en el campo de la incidencia sobre lo social. De otra parte, la crítica trabajada desde los 
propios campos disciplinares y los respectivos sustentos epistémicos, conduce a una forma de compartir las deliberaciones asociadas a la pertinencia de la explicación de lo social ofrecida por las diversas disciplinas. Los cambios sociales y culturales actualmente en curso y a velocidades diferente, mueve los ejes en los cuales la ciencia social apoyaba su planteamiento y dirimía de manera afirmativa.

Estos discursos a los cuales las ciencias sociales recurren, se encuentran tensionados por la propia actualización que las disciplinas han realizado. En la actualidad, la retórica como dimensión que explicaba los discursos, ha sido superada y criticada en los trabajos que posicionan justamente uno de los saltos cualitativos a los cuales se enfrenta esta categoría clásica (LAHIRE, 2016).

Siguiendo a Squella (2019), Hiernaux (2010) y Van Dyck (1996), los discursos condensan materialidades y simbólicas, por lo cual, los discursos producen subjetividades que trasuntan una forma contradictoria, confusa e inasible entre fundamentar la ciencia, la generación de conocimiento y su impacto en la forma de incidir en el pensamiento social. De manera principal si consideramos a este tipo de pensamiento, como un movimiento vital, en flujos de condicionamiento, crítico para advertir las posibilidades tanto por la afirmación como por la des-confirmación, es decir, el discurso como práctica situada que contiene la acción histórica (GIANNINI, 2016; ARENDT, 2016).

Las temporalidades por tanto, contribuyen a identificar los momentos en los cuales se producen rupturas en la ciencia social, como también en su producción científica. Las fisuras acontecidas en las disciplinas, evidentemente han recibido la influencia de lo social y, bajo esta lógica se han visto beneficiadas de una crítica que inevitablemente conduce a procesos de inflexión crítica, sobre el contenido y sobre lo procedimental.

Si auscultamos la presencia de las ciencias sociales inscritas en el tiempo social como larga duración, el quehacer de esta ciencia a través de quienes la construyen, se puede afirmar la juventud de la misma. Su presencia fue de importancia y generó fisura a la forma de pensamiento social de la época, no obstante, la existencia misma de la ciencia social posee atributos materiales, los cuales expresan al mismo tiempo -aunque no se aprecian-, la dimensión intangible que no puede ser obviada u omitida.

Las ciencias sociales en su devenir, como flujo a través del tiempo, han logrado sortear sustantivamente y en grados variables por cada disciplina, la subordinación inicial, con la cual se posicionaban frente a las ciencias básicas, como al mismo tiempo, por el estatuto y ordenamiento que realizaba la propia cultura científica respecto de aquellas. Una de las dis-continuidades y ruptura en lo que a fundamentos de las ciencias refiere, es la idea y valor asociada a la neutralidad de ésta, cuestión definida desde el positivismo, neopositivismo, cuyo protocolo y horizonte normativo logra instalarse de la misma forma en las ciencias sociales de forma transversal. Esta neutralidad o posicionamiento respecto al objeto de estudio y sus derivadas, más que una forma de procedimiento formal y técnico, fue una configuración de un pensamiento construido que informó y moldeó prácticas y lógicas que 
trascendieron incluso a la misma actividad científica.

La superación de esta inentendible neutralidad, fue plausible a propósito de la recuperación de otras corrientes asociadas a la filosofía de la ciencia, como también a la creación de nuevos enfoques que producto de la crítica de una lectura monocorde y unilateral hacia la realidad social, desplegaron todo el potencial elaborado desde el deconstruccionismo, como reflexión y acción deliberativa. En este espacio de recuperación y creación, vale la pena destacar que este pensamiento de inflexión crítica resulta justamente de la forma como las ciencias sociales interrogan su quehacer al interior de la sociedad y la validez que puede adquirir como ámbito que contribuye a explicar lo social, desde un enfoque histórico y en movimiento. Las nuevas miradas, lecturas sobre y desde lo social, asoman y se afirma a partir de las nuevas pistas que identifican las ciencias sociales con todos sus movimientos y envolvimientos que implican a quienes cultivan los oficios de las ciencias sociales, haciendo evidente la dialéctica entre compromiso y distancia (ELIAS, 2002).

\section{Las materialidades como expresiones de contornos y coacciones}

Los trabajos de Godelier, vinculados a la dialéctica entre lo ideal y lo material hacen plausible advertir la relación entre la práctica científica y la constitución de lo social. Las materialidades que definen la región latinoamericana, en su componente económico, político, social y cultural, generan una forma específica en la cual la reproducción social es una constante a través del tiempo (GODELIER, 1984). Las desigualdades estructurales van conformando el paisaje cultural en el cual se desenvuelve cada formación social, con sus particularidades, pero también con similitudes que hacen la pertenencia material y simbólica de esta región del mundo. Las ciencias sociales no quedan exentas de estas realidades específicas, lo cual agrega elementos necesarios de tener en cuenta, para la generación de conocimientos desde y sobre lo social. Los debates y controversias referidas a las modalidades en las cuales la actividad científica se liga a los desafíos locales, provoca la pertinencia de evidenciar la tensión permanente entre lo estructural y la experiencia cotidiana de los individuos, grupos y colectivos.

En este derrotero, la formación universitaria se ve expuesta a identificar los alcances de sus protocolos, la revisión de los programas y las modalidades como las ciencias sociales no pueden transformarse sólo en una caja de resonancia de la explicación de lo social en claves clásicas, sino en su contrario, como la propia actividad científica queda indefectiblemente ligada a los desafíos que se re-producen sistemáticamente, siendo evidente un ámbito de cambio social deliberativo.

El interés de las ciencias sociales en su componente de investigación, adquiere densidad cuando se interroga la dimensión referida a la intervención bajo una óptica densa y como acción histórica, relevando la intención de los horizontes normativos en proceso de revisión crítica. La noción de cambio social en claves interdisciplinarias incorporando aquellas 
ciencias fronteras y no fronteras, invitando a distintos actores y sujetos que se posicionan en niveles diferenciados, y relevando los espacios posibles y no posibles de la acción pública, gestan formas diversas en las cuales las ciencias sociales logran articular y sopesar la complejidad de lo social y su despliegue en los cuerpos individuales y sociales (LE BRETON, 2002).

Siguiendo esta lógica, las ciencias sociales en su devenir y en la expresión de éste considerando este pasado reciente -utilizando la nomenclatura del estudio de las memorias sociales y emblemáticas en América Latina-, sigue aportando esta lectura crítica alrededor de la producción de conocimientos, como una actividad situada que busca potenciar no sólo respuestas particulares a las hipótesis trabajadas, sino que al mismo tiempo, se dispone para identificar los debates, controversias, como espacios deliberativos y contenciosos que reflejan la urdimbre de lo social.

Esta acción reflexiva de las ciencias sociales sobre la incidencia en la dimensión de lo público, remite a esta dis-continuidad sucedida en el plano de una manera unívoca de generar conocimiento, pero de una forma más compleja afecta la propia idea y representación -citando nuevamente a Godelier-, respecto a la "intervención" como condición de posibilidad y de un posicionamiento que lo diferencia de otras ciencias, otorgando un valor adicional que prestigia y legitima desde otros referentes y encuadres. En cierta parte y tomando algunos resguardos necesarios, la intervención de las ciencias sociales se reflexiona desde las variabilidades y lo escalar, logrando desacomodar un posicionamiento de tipo canónico, más bien, opera en grados distintos y en sus contrarios, es decir, una disposición a modificar y configurar los oficios de las ciencias sociales en sus múltiples relaciones teóricas, metodológicas, epistémicas y políticas.

Interrogar la pertinencia de la intervención en su componente normativo bajo la lógica de los promedios sociales, fue una sugerente dis-continuidad facilitando la mirada crítica no sólo referida al control social, sino igualmente sobre la configuración del orden, como inquietud de lo político y la contribución que hacen los oficios y disciplinas hacia la configuración de la vida en común, como ideario posible (LECHNER, 2002).

Esta dis-continuidad, posibilitó una nueva lectura sobre la ciencia social y el ejercicio profesional específico, haciendo una nueva crítica reflexiva en la cual se diferencie la legalidad y la legitimidad de la acción social, cuestión que excede a las propias disciplinas de las ciencias sociales, pero que, igualmente la contiene.

Esta lectura de la dimensión estructural y de coacción en la cual se desarrollan las ciencias sociales, puede ser analizada tanto por el componente material que la delinea, como por la decodificación de los intangibles, lo cual puede colaborar en las dis-continuidades con las cuales se va apropiando de lo social. En esta acción científica y política a la vez, se logran observar los espacios que logran ser conquistados, como también se aquilatan los intersticios disciplinarios en los cuales asoman las disputas, como un ámbito de aquello que es más próximo. 
Revisar de manera de-formalizada algunas de las dis-continuidades generadas en este campo de la producción científica, posibilita superar en cierta parte, la tendencia a lo nuevo como acción mecánica, la sobrevaloración del presente social, la reiteración de una lógica obsesionada por el orden social, como horizonte normativo único y homogéneamente definido a través de los promedios sociales (FISHER, 2018).

En cierta síntesis preliminar, los fundamentos disciplinarios de las ciencias sociales, se vinculan al desarrollo y preguntas abiertas que las propias sociedades han provocado en el último tiempo (MARTUCCELLI, 2019). Los giros epistémicos de forma acumulativa, han posibilitado que las construcciones disciplinarias contemporáneas, se beneficie de las rupturas y de los puntos de saturación a los modos clásicos y canónicos (LAHIRE, 2017; BERTHELOT, 2000). En esta perspectiva, podemos distinguir entre aquellos ámbitos que configuran lo referido a la construcción del objeto y a los modos con los cuales se posibilita esta acción metodológica. De manera complementaria, se destacan aquellas lógicas que relevan debates y dilemas que son parte de los desafíos que asume la ciencia cuando se construye en términos que las controversias sean parte del desarrollo de éstas, no eximiendo a las ciencias sociales de esta forma sugerente de concretar la reflexividad como actividad humana (CHERNILO, 2011).

\section{Condiciones de posibilidades: las mentalidades como productoras de subjetividades}

Inevitablemente los movimientos de dis-continuidades implican la formación de mentalidades, a propósito de la interrogante referida a las perspectivas críticas en la construcción disciplinaria de las ciencias sociales.

Las mentalidades circulan como formas en las cuales se construyen ciertas lógicas y/o discursos que puede apropiarse de un tiempo que excede al aquí y alhora, pero que igualmente va preformando formas de estar y ocupar el espacio social. Se puede apreciar en esta lógica, ciertas tendencias que serían significativas de subrayar de manera de-centrada y de-formalizada:

a. Desde lo contingente, podemos identificar las formas en como lo situado ha colonizado una dimensión de carácter micro social de tal nivel de encapsulamiento, que ha vaciado y/o des-materializado la noción del individuo social, enfatizando lo individual hasta el borde del individualismo como orientación próxima al solipsismo social. Por antonomasia, esta afirmación del sujeto gira en una dirección que conduce de manera directa hacia lo que algunos autores definen la sociedad a escala del individuo. Los estudios de Karmy, pueden ayudarnos en esa pérdida de espesura de la alteridad, no sólo como vínculo entre individuos/sujetos/actores, sino la alteridad como las modalidades en las cuales se van delineando los espacios 
institucionales para la comunidad en el sentido de lo político, siendo la experiencia individual y colectiva la que trasuntan estas opciones (KARMY, 2016).

b. En una persistencia de larga duración, la noción de crítica tributada del análisis marxista clásico y sus producciones posteriores, sigue en ciertos casos una forma invariante, sin advertir aquellas rupturas que la propia producción marxista ha logrado identificar como objetos de crítica (BYUNG-CHUL, 2013; MOUFFE, 2007). EI modelo agonístico referido a la democracia producto de la acción deliberativa, precisa volver sobre lo efectivo y afectivo de lo político como sentido y núcleo principal, haciendo por lo mismo, que la política revise aquello contingente en claves complejas y superando las modalidades de militancias clásicas, las cuales en la actualidad se combinan en alteridades y en una re-definición de las categorías nosotros-ellos.

c. En una supuesta actualización de las ciencias sociales, se puede llegar a re-producir una lógica en la cual las ciencias sociales, remiten a una expresividad sistemática y reactiva frente a las modas intelectuales que va instalando la sociedad del espectáculo, aunque no sea el propósito deseado (GALENDE, 2017).

d. La industria cultural es una máquina que produce materialidades vinculadas al deseo, expresiones asociadas a la autoafirmación, tangibles que concretan la dimensión de lo peculiar, de aquello único, de lo definido como diferente, de los ámbitos que resaltan la distinción. La industria cultural como expresión de la cultura de la entretención, no es otra cosa que un montaje para construir una distracción sistemática y evitar atravesar conflictos, enfrentar controversias y/o bien sostener lo contencioso, como modo en el cual se condensa lo más próximo a la vida en común como 'vita activa'.

En síntesis, estas tendencias pueden ser reflexionadas en grados variables y a escalas diferentes. En sincronía, la crítica adquiere potencialmente valor y sentido, si el modo de producción es interrogado como movimientos que se des-montan en velocidades diferidas entre la materialidad, la simbólica y las nuevas formas de industria cultural instaladas en el actual tiempo social (FISHER, 2016).

Por tanto, desde las ciencias sociales y su vínculo a la sociedad que la produce y la contiene, pudiera formularse algunas interrogantes: ¿Qué soltamos de forma reflexiva y que preservamos? ¿Cómo logramos generar mentalidades que vayan siendo la expresión de la construcción socio-histórica que conserva, pero que no sea conservadora, o bien, cómo logramos otorgar nuevas complejidades a la idea de cambio social? ¿Cómo se logra apropiar la materialidad de lo social, como condición de posibilidad para generar nuevas rupturas en el quehacer científico?

Primavera 2019. 


\section{Referencias}

ARENDT, H. La Condición Humana. Paidós: Buenos Aires, 2016.

ARENDT, H. De la Historia a la Acción. Paidós: Buenos Aires, 1995.

BERTHELOT, J. M. Épistémologie d'une discipline. Textes fondamentaux. De Boeck Université: Bruxelles, 2000.

BRAUDEL, F. La Historia y las Ciencias. Alianza Editorial: Madrid, 1979.

BURKE, P. Escribiendo Historia en el Siglo 21: Desafíos y Respuestas. Ediciones Instituto de Historia, Pontificia Universidad Católica de Valparaíso: Valparaíso, 2019.

BYUNG-CHUL, H. La sociedad de la transparencia. Herder Editorial: Barcelona, 2013.

BYUNG-CHUL, H. La sociedad del cansancio. Herder Editorial: Barcelona, 2012.

CHERNILO, D. La Pretensión Universalista de la Teoría Social. LOM Ediciones: Santiago de Chile, 2011.

ELIAS, N. Compromiso y Distanciamiento. Editorial Península: Madrid, 2002.

FISHER, M. K-Punk Volumen 1 Escritos reunidos e inéditos (libros, películas y televisión). Caja Negra: Buenos Aires, 2018.

FISHER, M. Realismo Capitalista ¿No hay alternativa?. Caja Negra: Buenos Aires, 2016.

GALENDE, F. Diplomado Crítica de Arte Contemporáneo Chileno. Pontificia Universidad Católica de Valparaíso: Chile, 2017.

GIANNINI, H. La 'reflexión' cotidiana. Hacia una arqueología de la experiencia. Revista Filosofía Universidad de Chile: Santiago de Chile, 2016.

GODELIER, M. L'idéel et le matériel. Fayard: France, 1984.

GOICOVIC, I. Cátedra Perspectivas Históricas - Programa Magister Pontificia Universidad Católica de Valparaíso: Valparaíso, Chile, 2019.

HALBWACHS, M. Les cadres Sociaux de la Mémoire. Presses Universitaire de France: Paris, 1952.

HIERNAUX, J. P. Analyse Structurelle. Université Catholique de Louvain: Bélgique, 2010.

HOBSBAWM, E. Gente poco corriente: Resistencia, rebelión y jazz. Editorial Crítica: Barcelona, 2013.

JELIN, E. Los trabajos de la memoria. Siglo XX Editores: España, 2002.

KARMY, R. Escritos bárbaros. LOM Ediciones: Santiago de Chile, 2016.

LAHIRE, B. Pour la Sociologie. La Découverte, Paris, 2016.

LE BRETON, D. Antropología del Cuerpo y Modernidad. Ediciones Nueva Visión: Buenos Aires, 2002.

LECHNER, N. Las sombras del mañana. LOM Ediciones: Santiago de Chile, 2002.

MARTUCCELLI, D. Cambio de rumbo. LOM Ediciones: Santiago de Chile, 2007.

MOUFFE, C. En torno a lo político. Fondo de Cultura Económica: Madrid, 2007. 
RANCIÉRE, J. El reparto de lo sensible. LOM Ediciones: Santiago de Chile, 2009.

SIMMEL, G. Sobre la Individualidad y las Formas sociales. Escritos Escogidos. Universidad Nacional de Quilmes: Argentina, 2002.

SQUELLA, A. Democracia. ¿Crisis, decadencia o colapso?. Editorial Universidad de Valparaíso: Valparaíso, 2019.

VAN DYCK, T. Las Estructuras y Funciones del Discurso. Siglo XXI editores, México, 1996.

WALLERSTEIN, I. Las incertidumbres del saber. Editorial Gedisa: Barcelona, 2009.

Recebido em: 04/11/2019.

Aceito em: 16/02/2020. 